\title{
Influence of Maxillary Central Incisor Asymmetries on the Perception of Dentofacial Esthetics at Repose
}

\author{
Ludmilla Macedo Bacelar ${ }^{1}$, Gabriela Meyge de Brito², Paulo Sergio Flores Campos ${ }^{3}$, Cristina \\ Cangussu ${ }^{4}$, Diana Simoes ${ }^{2}$, Andre Wilson Machado ${ }^{5 *}$

\begin{abstract}
${ }^{1}$ Undergraduation student, Federal University of Bahia, Salvador, Bahia, Brazil.
${ }^{2}$ Doctorate degree student, Dentistry and Health section, Federal University of Bahia, Salvador, Bahia, Brazil.

${ }^{3}$ Associate professor, Department of Oral Radiology, Federal University of Bahia, Salvador, Bahia, Brazil.

${ }^{4}$ Associate professor, Departament of Social and Pediatric Dentistry, Federal University of Bahia, Bahia, Brazil.

${ }^{5}$ Associate professor, Department of Orthodontics, Federal University of Bahia, Salvador, Bahia, Brazil. awmachado@gmail.com

*Corresponding Author: Andre Wilson Machado, Associate professor, Department of Orthodontics, Universidade Federal da Bahia, Brazil.
\end{abstract}

\begin{abstract}
Background: Dentofacial esthetic has been a growing quest in contemporary society. The aim of this study was to evaluate the influence of maxillary central incisor edge asymmetries on the perception of dentofacial esthetics at reposeamong orthodontists and laypersons. Four facial frontal photographs at repose were selected, of a White man and woman; and of an African Descendent man and woman, between 25 and 30 years of age. The images were digitally altered to create asymmetries (tooth wear) in the left maxillary central incisor in increments of $0.5 \mathrm{~mm}$ and $1.0 \mathrm{~mm}$. After a pilot study and sample calculation, the final images were randomly assembled in a photograph album that was evaluated by 47 orthodontists and 47 laypersons. Each evaluator was asked to assess the attractiveness of the images individually, with the use of visual analog scales. The data collected were statistically analyzed, with the Analysis of Variance with Tukey post-test, and the unpaired Student'st test.
\end{abstract}

Results: The most attractive images were those without asymmetries, and the most unattractive were those with asymmetries of $1.0 \mathrm{~mm}$. In general, for both groups of evaluators, the larger the asymmetry was, the less attractive were the images.

Conclusion: The presence of maxillary central incisors edges asymmetries directly affected the dentofacial esthetic appearance at repose.

Keywords: Dental esthetics, Asymmetry, Orthodontics.

\section{BACKGROUND}

In contemporary society, there has been a growing quest for dentofacial esthetics that has increased the number of patients seeking esthetic dental treatments. For this reason multidisciplinary treatments are extremely important for helping professionals to attain the satisfaction of their patients. ${ }^{1}$

With the goal of obtaining ideal esthetic results, it is imperative to establish parameter references.
Over the course of many years, clinical opinions were responsible for esthetic guidance in dental treatments. ${ }^{1,2}$ However, these parameters could be questioned, because the concept of beauty is subjective and is influenced by different factors such as culture, social status and educational level. .,3-6 $^{13}$

The literature has shown that among the references used for obtaining attractive smiles, the presence of symmetrical maxillary central incisors edges is of fundamental importance ${ }^{1,7,8}$. According to Chiche et 
al. ${ }^{7}$ the need for symmetry is more relevant closer to the midline, while slight asymmetries distant from the midline are acceptable. Therefore, variations in color, shape, height and width at the central incisors are considered critical. According to this concept, incisal and/or gingival asymmetries at the central incisors need to be carefully evaluated. According to the literature ${ }^{8,9}$ any level of incisal asymmetries between maxillary central incisors is considered unattractiveand must be corrected in order to achieve optimal esthetic results.

Although various studies have researched the esthetic characteristics of the smile, there is a need to focus attention on studies with the esthetics at repose and esthetics during speech. This has become fundamental, because in many day-to-day situations, esthetics is evaluated when persons are in situations with the face in the repose position, and during interpersonal communication. One research showed that the amount of exposure of the maxillary incisors in the rest position is the most important characteristic for patients' satisfaction with their dentofacial esthetics, both in women and men. ${ }^{10}$

After analyzing the above-mentioned information, several questions arose. If an asymmetry is related to the maxillary incisor edges, what do laypeople and orthodontists perceive when evaluating photographs at repose? In other words, what is the threshold for these evaluators when analyzing uneven central incisors at repose? Considering the absence of studies regarding this topic, and taking into account that incisal asymmetries in central incisors during the smile are highly deleterious from the esthetic point of view, ${ }^{8,9}$ the aim of this study was to evaluate the influence of maxillary central incisor edge asymmetries on the perception of dentofacial esthetics at repose among orthodontists and laypersons.

\section{METHODS}

This study was approved by the Dental School Research Ethics Committee, Protocol No.2.072.112, and registered by the National Research Ethics Committee, No. 64405016.0.0000.5024. The individuals photographed signed a Term of Free and Informed Consent.

The sample size was determined by means of a pilot study, in which the Student's- $t$ test was applied, with a power of $80 \%$, and bilateral alpha test level of $5 \%$. The determination was that the sample had to include at least 47 peoplein each group of evaluators.

To conduct this research, four facial frontal photographs taken at repose were selected, of an African descendent man and woman; and of a White man and woman, between 25 and 30 years of age. The individuals presented adequate facial symmetry, good proportion between the facial thirds and presented $2.5 \mathrm{~mm}$ of maxillary central incisor exposure ${ }^{11}$.

Images selected were digitally altered by using the program Adobe ${ }^{\circledR}$ Photoshop ${ }^{\circledR}$ CS4 (Seattle, WA, U.S.A.), with the purpose of reproducing symmetrical images, enhancing the color, brightness and contrast, in addition to removing any discolorations in skin and lips. ${ }^{1,2,9,11}$ After this, from the original image, three images were created for each of the individual chosen, with the following characteristics between the incisal edges of the central incisors: perfect symmetry; 0.5 $\mathrm{mm}$ of asymmetry, and $1.0 \mathrm{~mm}$ of asymmetry (Figs. $1,2,3$ and 4).
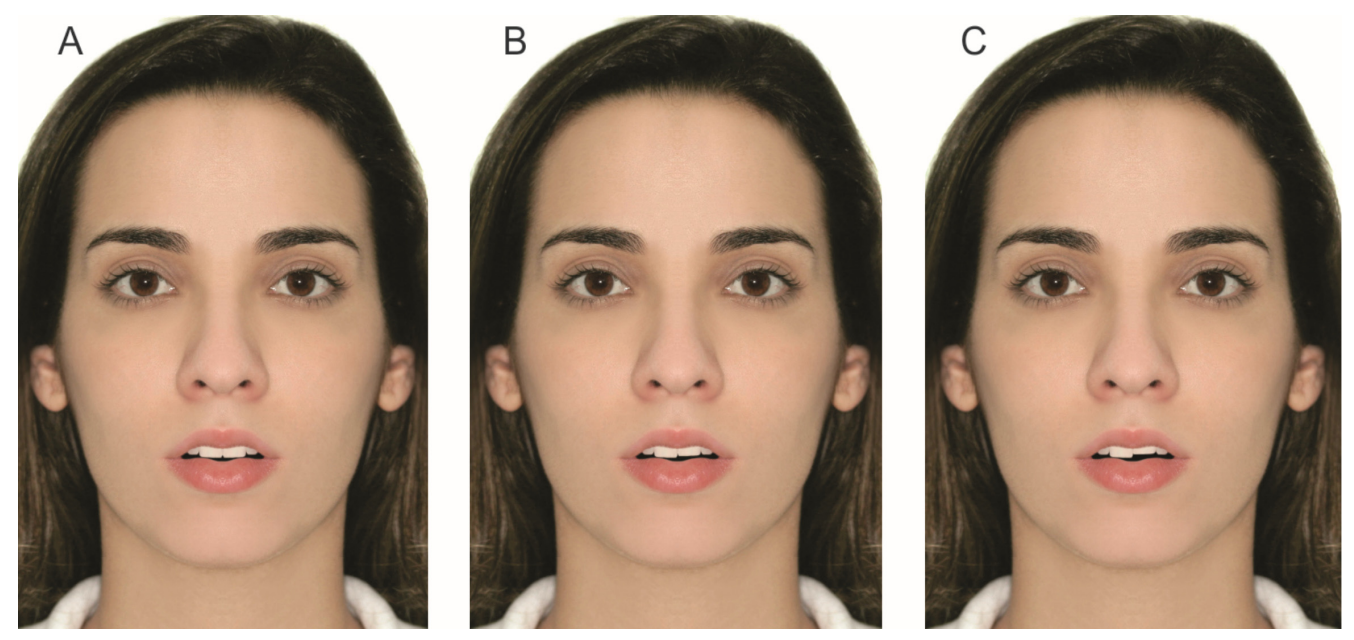

Fig 1. Full-face African descendent woman in 0.5-mm increments: A, control; $B, 0.5 \mathrm{~mm}$ asymmetry of central incisor; $C, 1.0 \mathrm{~mm}$ asymmetry of central incisor. 

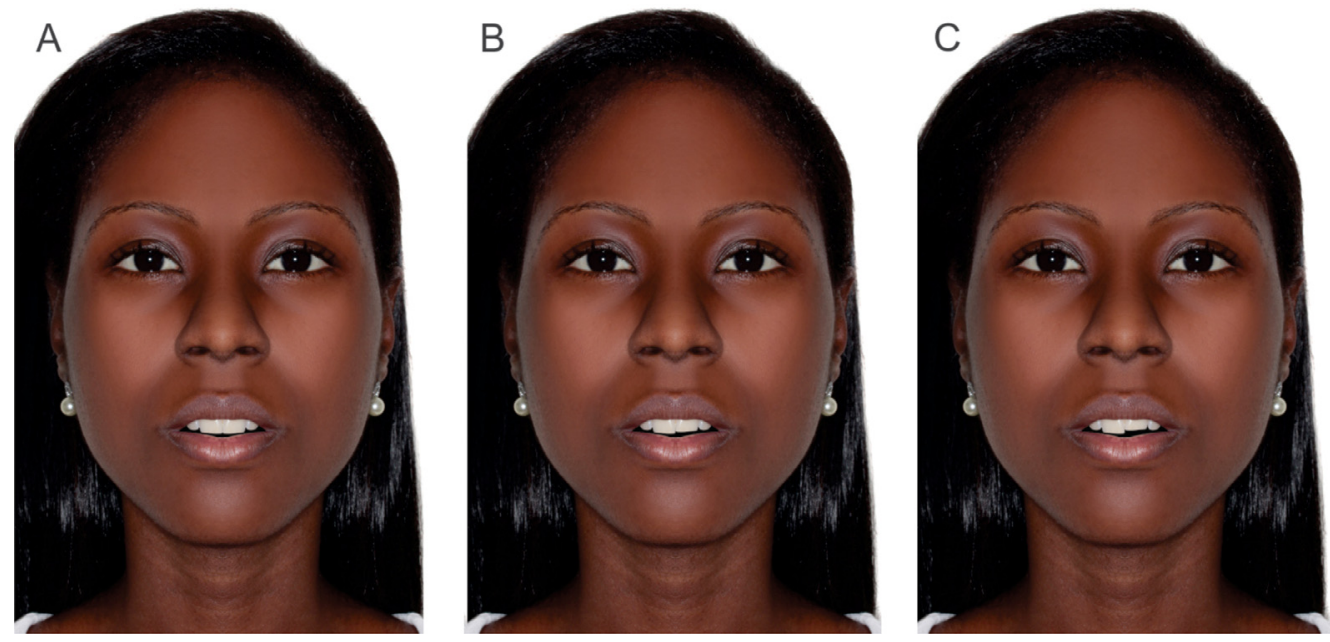

Fig 2. Full-face White woman in 0.5-mm increments: A, control; $B, 0,5 \mathrm{~mm}$ asymmetry of central incisor; $C, 1.0$ mm asymmetry of central incisor.
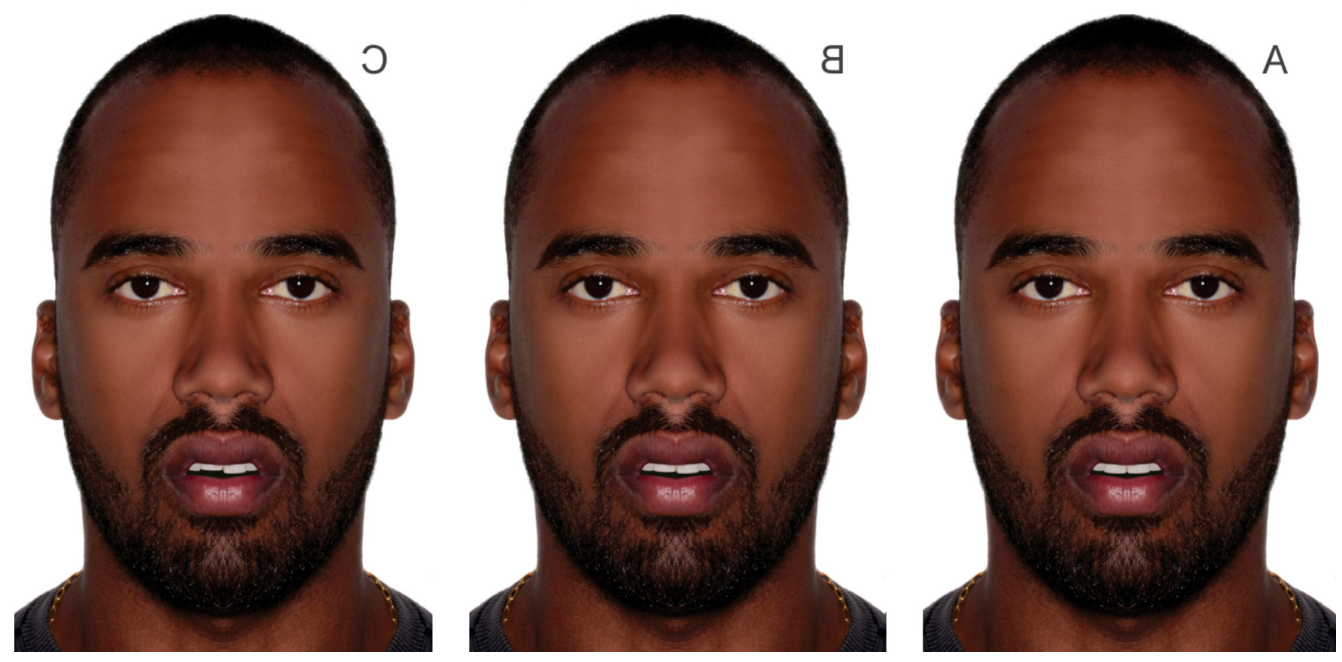

Fig 3. Full-face African descendent male in 0.5-mm increments: A, control; $B, 0,5 \mathrm{~mm}$ asymmetry of central incisor; $C, 1.0 \mathrm{~mm}$ asymmetry of central incisor.
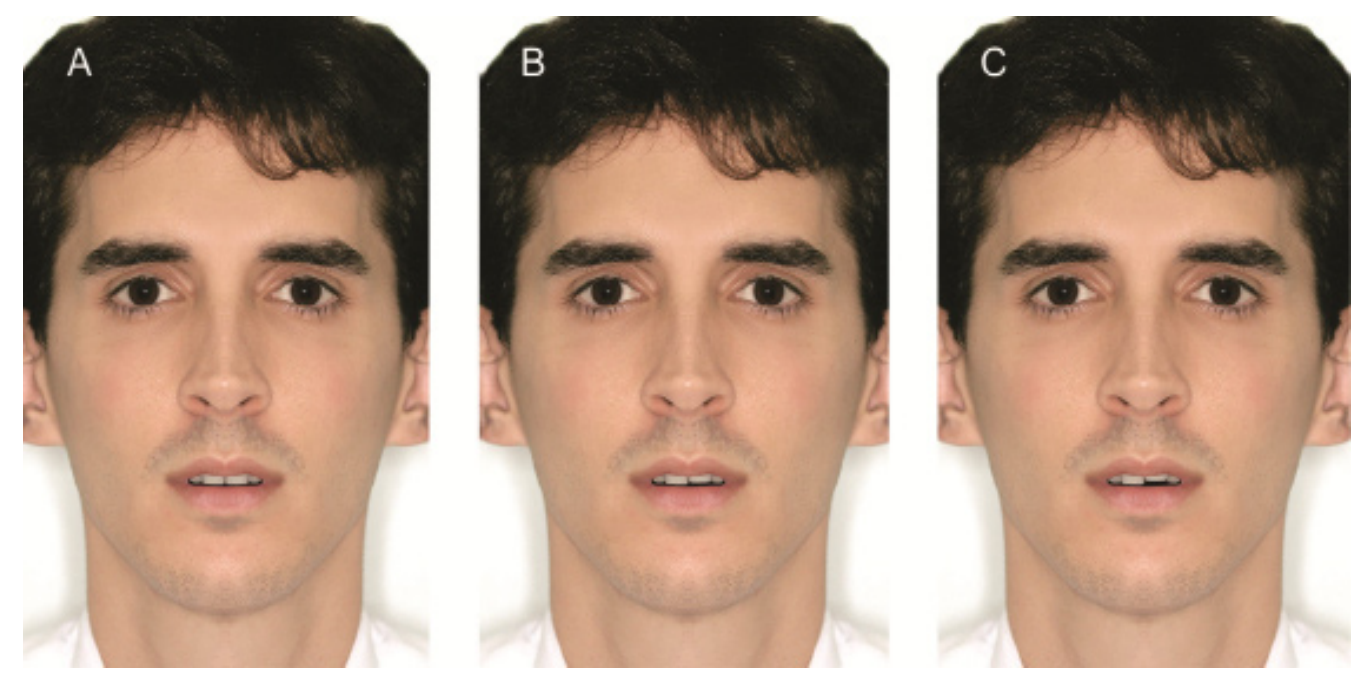

Fig 4. Full-face White male in 0.5-mm increments: $A$, control; $B, 0,5 \mathrm{~mm}$ asymmetry of central incisor; $C, 1.0 \mathrm{~mm}$ asymmetry of central incisor 
The top limit of the images was the region a little above the top of the head, and the bottom limit, the base of the neck. ${ }^{5,11-15}$ To guarantee calibration and magnification of the images, every millimeter measured in the image at the computer screen and at the image printed was equivalent to every millimeter clinically measured on the patient, using the width of the right maxillary central incisor as reference. ${ }^{9,11}$

Final images were printed and randomly assembled in a photo album containing 12 pages. The album was given to the 94 raters (47 orthodontists and 47 laypersons with a college education but no dental background). Information with reference to the study was explained to every evaluator, and they were asked to evaluate each image individually, according to the attractiveness of the dentofacial esthetic appearance. Together with the album, they were also provided with a printed simulation of 12 rulers (visual analog scale VAS), representing one for each image. This was a 100 $\mathrm{mm}$ long ruler, without grading with the following descriptors at the extremities: "very unattractive" on the extreme left, and "very attractive" on the extreme right. In the middle, there was the midpoint of the sale, providing a line of reference for the average level of attractiveness. ${ }^{2,9,14,15}$

All the evaluators were instructed to mark a point along the scale, according to the level of attractiveness associated with each image. The scores were then measured in millimeters with use of an electronic digital caliper (Starrett, Suzhou, China).

To evaluate the reliability of the method, 12 evaluators from each group were randomly selected. They were invited to evaluate a series of images of one of the individuals, in which there were two identical images. ${ }^{8,9,11}$ The intra-class test was used to compare the scores awarded to these images, so that it was possible to determine whether there was agreement between the scores. The result of 0.77 showed good reliability of the method.

Descriptive statistics were reported as means and standard deviations. To make comparisons between the images with different incisal asymmetries, oneway analysis of variance with the Tukey post-test were used; and to compare the distribution of mean scores between orthodontists and laypersons, the unpaired Student'st test was used. The level of significance was established at $5 \%$.

\section{RESULTS}

Tables 1 and 2 demonstrate the results obtained in the evaluation of the African descendent and White woman. For both women the best scores were attributed to the symmetrical images, while the least attractive was the image with asymmetry of $1.0 \mathrm{~mm}$ $(\mathrm{P}<.001)$. These results coincided for the two groups of evaluators.

In the evaluations made of the African descendent and White man, the same behavior as previously described was observed (Tables 3 and 4).

When the scores were compared between the orthodontists and laypersons, no statistically significant differences were found in most situations (8 out of 12 analyses) ( $P>0.05$ ). In the four situations where there were differences, laypersons awarded higher scores than the orthodontists $(\mathrm{P}<0.05)$. (Tables 1 to 4$)$.

Table 1. Means and standard deviations of the attractiveness of the images in full-face views of White woman for the group of orthodontists and laypersons. SD, standars deviation; Orth x LP, Orthodontists x laypersons.

\begin{tabular}{|c|c|c|c|c|c|c|c|c|}
\hline \multicolumn{9}{|c|}{ White Woman } \\
\hline & & \multicolumn{3}{|c|}{ ORTHODONTISTS } & \multicolumn{3}{|c|}{ LAYPERSONS } & \multirow[b]{2}{*}{ ORTH X LP (P) } \\
\hline & & Mean & SD & Results & Mean & SD & Results & \\
\hline A & 0 & 64.1 & 19.74 & \multirow{3}{*}{$\mathrm{A}>\mathrm{B}>\mathrm{C}$} & 71.16 & 19.04 & \multirow{3}{*}{$A>B>C$} & 0.081 \\
\hline B & $0.5 \mathrm{~mm}$ & 29.13 & 16.75 & & 41.09 & 21.29 & & 0.003 \\
\hline $\mathrm{C}$ & $1.0 \mathrm{~mm}$ & 13.05 & 14.36 & & 23.43 & 15.71 & & 0.001 \\
\hline
\end{tabular}

Table 2. Means and standard deviations of the attractiveness of the images in full-face views of African Descendent woman for the group of orthodontists and laypersons. SD, standars deviation; Orth x LP, Orthodontists x laypersons.

\begin{tabular}{|c|c|c|c|c|c|c|c|c|}
\hline \multicolumn{9}{|c|}{ African Descendent Woman } \\
\hline & & \multicolumn{3}{|c|}{ ORTHODONTISTS } & \multicolumn{3}{|c|}{ LAYPERSONS } & \\
\hline & & Mean & SD & Results & Mean & SD & Results & ORTH X LP (P) \\
\hline A & 0 & 70.6 & 21.59 & \multirow{3}{*}{$A>B>C$} & 70.8 & 19.13 & \multirow{3}{*}{$A>B>C$} & 0.964 \\
\hline $\mathrm{B}$ & $0.5 \mathrm{~mm}$ & 29.94 & 20.15 & & 34.85 & 17.37 & & 0.208 \\
\hline $\mathrm{C}$ & $1.0 \mathrm{~mm}$ & 14.77 & 15.23 & & 23.31 & 17.51 & & 0.013 \\
\hline
\end{tabular}


Influence of Maxillary Central Incisor Asymmetries on the Perception of Dentofacial Esthetics at Repose

Table 3. Means and standard deviations of the attractiveness of the images in full-face views of White man for the group of orthodontists and laypersons. SD, standars deviation; Orth x LP, Orthodontists x laypersons.

\begin{tabular}{|c|c|c|c|c|c|c|c|c|}
\hline \multicolumn{9}{|c|}{ White Man } \\
\hline & & \multicolumn{3}{|c|}{ ORTHODONTISTS } & \multicolumn{3}{|c|}{ LAYPERSONS } & \\
\hline & & Mean & SD & Results & Mean & SD & Results & ORTH X LP (P) \\
\hline $\mathrm{A}$ & 0 & 61.99 & 21.77 & \multirow{3}{*}{$A>B>C$} & 21.77 & 17.76 & \multirow{3}{*}{$A>B>C$} & 0.567 \\
\hline $\mathrm{B}$ & $0.5 \mathrm{~mm}$ & 32.5 & 20.1 & & 20.1 & 20.04 & & 0.117 \\
\hline $\mathrm{C}$ & $1.0 \mathrm{~mm}$ & 15.7 & 14.9 & & 14.9 & 16.82 & & 0.055 \\
\hline
\end{tabular}

Table 4. Means and standard deviations of the attractiveness of the images in full-face views of Afro-descendant man for the group of orthodontists and laypersons. SD, standars deviation; Orth x LP, Orthodontists $x$ laypersons.

\begin{tabular}{|c|c|c|c|c|c|c|c|c|}
\hline \multicolumn{9}{|c|}{ African Descendent Man } \\
\hline & & \multicolumn{3}{|c|}{ ORTHODONTISTS } & \multicolumn{3}{|c|}{ LAYPERSONS } & \\
\hline & & Mean & SD & Results & Mean & SD & Results & ORTH X LP (P) \\
\hline$A$ & 0 & 52.74 & 22.34 & \multirow{3}{*}{$\mathrm{A}>\mathrm{B}>\mathrm{C}$} & 65.33 & 20.09 & \multirow{3}{*}{$A>B>C$} & 0.005 \\
\hline $\mathrm{B}$ & $0.5 \mathrm{~mm}$ & 28.99 & 15.87 & & 41.08 & 18.53 & & 0.001 \\
\hline $\mathrm{C}$ & $1.0 \mathrm{~mm}$ & 17.77 & 15.6 & & 24.15 & 16.82 & & 0.055 \\
\hline
\end{tabular}

\section{Discussion}

This study followed the trend of other published studies in the literature, ${ }^{8,9,11}$ but instead of evaluating the esthetic perception of the smile, the dentofacial esthetics at repose position was evaluated, by assessing different levels of incisal edges asymmetries between maxillary central incisors. The need to study the esthetics perception at repose is of great relevance, considering that dentofacial esthetics involve the smile, speech and repose. ${ }^{1}$ The latter analysis has become increasingly important, seeing that this position of the mouth is commonly used in folders, magazine covers, marketing of beauty products, among other purposes, and is associated not only with beauty, but also with sensuality and joviality. ${ }^{16,17}$ No studies that evaluated the presence of asymmetries in maxillary central incisors in this position were found, which makes this an unpublished study.

Our results showed that this aspect significantly affected the perception of dentofacial esthetics. Both orthodontists and laypersons attributed higher scores to the symmetrical images. Whereas, images with asymmetry of 0.5 and $1.0 \mathrm{~mm}$ received lower scores. The studies conducted by Ribeiro et al. ${ }^{8}$ and Machado et al. ${ }^{9}$ corroborates our findings when they found that in analyses of photographs of the smile, even small asymmetries of $0.5 \mathrm{~mm}$ in maxillary central incisors were considered unattractive by both laypersons and orthodontists. This reinforces the hypothesis that the closer to the midline, the greater the need for symmetry. $1,7,18$
It is worthy to emphasize that the data found in this study apply to patients who present some level of maxillary incisors exposure at repose, thus they are more directed towards young individuals. The amount of teeth exposure used in our study was based on the data found by Caramello et al. ${ }^{11}$ who showed that the most attractive dentofacial esthetics were found in photographs with incisal exposure of $2.0 \mathrm{~mm}$ and 2,5 $\mathrm{mm}$ at repose. Considering these values, asymmetries of 0.5 and $1.0 \mathrm{~mm}$ were created. No asymmetries exceeding $1.0 \mathrm{~mm}$ were created, because previous studies have demonstrated that extreme asymmetries were extremely unattractive. ${ }^{8,9}$ As the present study used esthetics perception scores from young individuals, further studies are necessary to answer the question of whether these results would apply to sample of older patients.

This study was conducted using four individuals, a White man and woman, and an African Descendent man and woman, with the purpose of broadening the findings and discussion about the topic. Therefore, the results showed that irrespective of gender and race, the presence of incisal asymmetry of the maxillary central incisors negatively influenced the perception of dentofacial esthetics at repose. When comparing our findings to other studies that evaluated the influence of asymmetries on smile esthetics perception, it was found a similar pattern of attractiveness when African descendent persons were compared towhite persons. ${ }^{9,15}$ Based on those data wehypothesize that when men and women, white and African descendent persons were compared, there appeared to be a convergence of the esthetic parameters. 
With the results of this research in mind, the orthodontist should give special attention in clinical situations of maxillary central incisors edges asymmetries. These problems might be caused by fracture, abrasion, compensatory eruption, and vertical error in bracket positioning among others. ${ }^{1,7,9}$ Our data emphasizesthe need for multidisciplinary treatments to achieve maximum symmetry between maxillary central incisors. Moreover, previous studies ${ }^{8,9}$ suggests that orthodontic treatment should focus more on incisor edges alignment than on gingival margin alignment since maxillary central incisors asymmetries of 0.5 $\mathrm{mm}$ are more commonly considered unaesthetic than gingival margin asymmetries of $1.5 \mathrm{~mm}$, as reported in the literature ${ }^{15}$.

The present study used a group of orthodontists as evaluators, because previous studies have shown that they are more critical, technical and sensitive in the perception of deviations from the ideal. ${ }^{8,9,13-15,19,20} \mathrm{~A}$ group of layperson evaluators, who represent the main users of dental services, were also used. The literature shows studies where no difference was found between the two groups, and others where there was differences, in which orthodontists were more critical than laypersons. In our study, in most situations there was no statistically significant difference between the two groups of evaluators (Tables 1 and 4).

Finally, it is important to remember that because thisstudy used computer-manipulated facial images at repose fromfour individuals and the opinion of specific groups ofindividuals, the results should be carefully analyzed. According to Kokich et al., ${ }^{21}$ when results and conclusions are based on mean values, the information must not be generalized for all patients, because esthetic appearance is subjective. Therefore, despite of the great importance of those findings to professionals to use as a basis for conducting treatment and guiding patients, individualization of treatments is always the most favorable option for routine clinical attendance.

\section{ConCLUSIONS}

The outcomes of this study demonstrate the following:

- The presence of asymmetries between maxillary central incisors edges has a deleterious influence on dentofacial esthetics perception at repose.
- According to orthodontists and laypersons, the most attractive images were those without asymmetry between maxillary central incisors edges. Any type of asymmetry in the central incisors edges ( 0.5 and $1.0 \mathrm{~mm}$ ) was evaluated as unattractive.

- When the mean scores between orthodontist and laypersons were compared, there was no statistically significant difference in most situations, and when there was difference, laypersons attributed higher scores than orthodontists.

\section{REFERENCES}

[1] Machado AW. 10 commandments of smile esthetics. Dental Press Journal of Orthod., 2014; 19: 136-57.

[2] Machado AW. The importance of maxillary central incisors symmetry on smile esthetics. Rev Clin Ortod Dent Press., 2014; 13: 40-51.

[3] McLeod C, Fields HW, Hechter F, Wiltshire W, Rody W, Jr., Christensen J. Esthetics and smile characteristics evaluated by laypersons. Angle Orthod., 2011;81:198-205.

[4] Kokich VO, Jr., Kiyak HA, Shapiro PA. Comparing the perception of dentists and lay people to altered dental esthetics. J Esthet Dent., 1999; 11: 311-24.

[5] Heravi F, Rashed R, Abachizadeh H. Esthetic preferences for the shape of anterior teeth in a posed smile. Am J Orthod Dentofacial Orthop., 2011; 139: 806-14.

[6] Cotrim ER, Vasconcelos ÁV, Haddad ACSS, Reis SAB. Perception of adults' smile esthetics among orthodontists, clinicians and laypeople. Dent Press J of Orthod., 2015;20:40-44.

[7] Chiche JG, Pinault A. Esthetics of anterior fixed prosthodontics/Gerard Chiche, Alain Pinault. 1994.

[8] Ribeiro J, Figueiredo B, Machado A. Does the presence of unilateral maxillary incisor edge asymmetries influence the perception of smile esthetics? J Esthet Restor Dent., 2017; 29: 291-97.

[9] Machado AW, Moon W, Gandini LG, Jr. Influence of maxillary incisor edge asymmetries on the perception of smileestheticsamongorthodontists and laypersons. Am J Orthod Dentofacial Orthop., 2013;143:658-64. 
[10] Zagar M, Knezovic Zlataric D. Influence of esthetic dental and facial measurements on the Caucasian patients' satisfaction. J Esthet Restor Dent., 2011; 23: 12-20.

[11] Caramello F, Bittencourt MAV, Machado AW. Influence of maxillary incisor level of exposure on the perception of dentofacial aesthetics among orthodontists and laypersons. J of the $\mathrm{W}$ Fed of Orthod., 2015; 4: 108-13.

[12] Parekh SM, Fields HW, Beck M, Rosenstiel S. Attractiveness of variations in the smile arc and buccal corridor space as judged by orthodontists and laymen. Angle Orthod., 2006; 76: 557-63.

[13] Rodrigues Cde D, Magnani R, Machado MS, Oliveira OB. The perception of smile attractiveness. Angle Orthod., 2009; 79: 634-9.

[14] Suzuki L, Machado AW, Bittencourt MAV. Avaliação da influência da quantidade de exposição gengival na estética do sorriso. Dental Press J of Orthod., 2011; 16: 1-10.

[15] Correa BD, Vieira Bittencourt MA, Machado AW. Influence of maxillary canine gingival margin asymmetries on the perception of smile esthetics among orthodontists and laypersons. Am J Orthod Dentofacial Orthop., 2014; 145: 55-63.
[16] Vig RG, Brundo GC. The kinetics of anterior tooth display. J Prosthet Dent 1978; 39:502-4.

[17] Desai S, Upadhyay M, Nanda R. Dynamic smile analysis: changes with age. Am J Orthod Dentofacial Orthop., 2009; 136: 310. e1-10; discussion 10-1.

[18] Lombardi RE. The principles of visual perception and their clinical application to denture esthetics. J Prosthet Dent., 1973;29:358-82.

[19] Pinho S, Ciriaco C, Faber J, Lenza MA. Impact of dental asymmetries on the perception of smile esthetics. Am J Orthod Dentofacial Orthop., 2007; 132:748-53.

[20] Krishnan V, Daniel ST, Lazar D, Asok A. Characterization of posed smile by using visual analog scale, smile arc, buccal corridor measures, and modified smile index. Am J Orthod Dentofacial Orthop., 2008;133:515-23.

[21] Kokich VO, Kokich VG, Kiyak HA. Perceptions of dental professionals and laypersons to altered dental esthetics: asymmetric and symmetric situations. Am J Orthod Dentofacial Orthop., 2006; 130:141-51.

Citation: Ludmilla Macedo Bacelar, Gabriela Meyge de Brito, Paulo Sergio Flores Campos, Cristina Cangussu, Diana Simoes, Andre Wilson Machado. Influence of Maxillary Central Incisor Asymmetries on the Perception of Dentofacial Esthetics at Repose. Archives of Dentistry and Oral Health. 2019; 2(2): 04-10.

Copyright: (c) 2019 Ludmilla Macedo Bacelar, Gabriela Meyge de Brito, Paulo Sergio Flores Campos, Cristina Cangussu, Diana Simoes, Andre Wilson Machado. This is an open access article distributed under the Creative Commons Attribution License, which permits unrestricted use, distribution, and reproduction in any medium, provided the original work is properly cited. 